Proceedings of Magneto-Optical Recording International Symposium '92, J. Magn. Soc. Jpn., Vol. 17, Supplement No. S1 (1993), pp. 201-204 (C) 1993 by The Magnetics Society of Japan

\title{
SUPER RESOLUTION READOUT OF A MAGNETO-OPTICAL DISK WITH AN IN-PLANE MAGNETIZATION LAYER
}

\author{
Y.Murakami, N.Iketani, J.Nakajima, A.Takahashi, K.Ohta, T.Ishikawa \\ Corporate Research and Development Group, Sharp Corporation \\ 2613-1, Ichinomoto-cho, Tenri, Nara 632, Japan
}

\begin{abstract}
A new magneto-optical (MO) disk, which consists of magnetic double layers, for super resolution readout was developed. We developed a new characteristic GdFeCo film as a readout layer whose magnetization direction changes from in-plane to perpendicular as a temperature rises. Using such a GdFeCo film combined with a DyFeCo recording film, higher linear density and track density than those of the conventional MO disks were achieved without an external magnetic field for readout and with a conventional optical head.
\end{abstract}

KEYWORDS : SUPER RESOLUTION, MAGNETO-OPTICAL DISK, IN-PLANE MAGNETIZATION

\section{INTRODUCTION}

To achieve higher density in magneto-optical (MO) disks, magnetically induced super resolution (MSR) readout methods were already proposed [1]. Two detection methods for MSR, FAD (front aperture detection) and RAD (rear aperture detection), were reported. Both methods utilize the temperature distribution of magnetic films in a light beam spot when the readout beam irradiates. For instance, recorded information can be read out only from the higher temperature region of a readout layer which corresponds to a front part of a readout beam spot in the FAD and a rear part of it in the RAD. In both methods, one or two external magnetic fields are necessary for the super resolution readout to make an optically masked area where all the magnetization direction of the readout layer was aligned either upward or downward.

We developed a new MO disk which enables the super resolution readout without an external magnetic field for readout. The new disk chiefly consists of double exchange-coupled magnetic layers including a new type of a readout layer. The feature of the readout layer is that its magnetization direction is in-plane at a room temperature and gets perpendicular at high temperatures. Therefore, when the readout laser beam irradiates, the polar Kerr effect occurs only at the higher temperature region of the readout layer, which corresponds to the central part of the light beam, and recorded information in a recording layer can be read out. This new technique utilizes only the temperature distribution of a medium and neither an initializing magnetic field nor a readout one are necessary for readout in this technique.

In this paper, a readout mechanism of the new medium and the static and dynamic characteristics are discussed.

\section{READOUT MECHANISM}

The readout mechanism of a new medium that we have developed is explained in figure 1 . The medium consists of magnetic double layers both of which are rare-earth (RE) and transition metal (TM) amorphous alloy films. In this medium, the recording layer is a conventional MO recording film, that means the magnetization direction is perpendicular to the film plane at from a room temperature to the Curie temperature. On the other hand, the magnetization direction of the readout layer is in-plane at the room temperature and gets

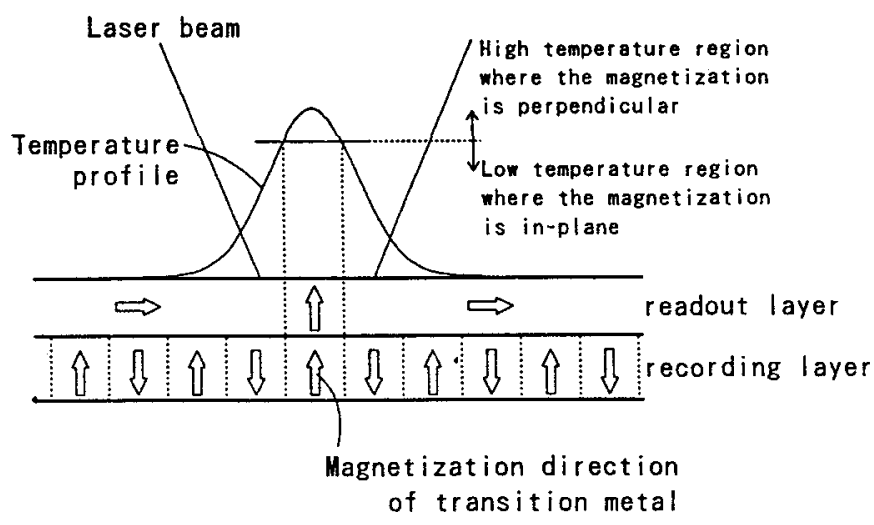

Fig. 1 Schenatic diagran of readout mechanisn in this study. 
perpendicular at high : temperatures. If the switched temperature region where the magnetization direction changes from in-plane to perpendicular is narrow enough, super resolution readout can be achieved with the readout mechanism explained as follows.

When a readout laser beam spot collimated by an objective lens irradiates the medium, the temperature distribution profile of the medium is like gaussian and the central part of the beam spot becomes hotter than the other around area. The magnetization direction of the readout layer changes perpendicular only at the hotter area and the polar Kerr effect occurs. At this moment, the magnetization direction in the recording layer is copied to the readout layer by the exchange coupling force. On the other hand, the around area outside the central area keeps the magnetization direction in-plane and does not exhibit the polar Kerr effect. Consequently, recorded information in the recording layer is optically masked by inplane magnetization of the readout layer and can be read out only at the central part of the beam spot. Afterwards, when the readout beam moves along with the rotating disk, the temperature of the previous readout area decreases and the magnetization direction of the readout layer changes back to in-plane. Thus, the area no longer exhibits the polar Kerr effect and the mark that is previously read hardly interferes the readout of coming recorded marks.

\section{EXPERIMENTS}

\section{Magneto-Optical Properties}

We utilized a GdFeCo film as the readout layer and a DyFeCo film as the recording layer. Typical magnetic properties of each layer are shown in Table 1. The RE-TM film, whose composition is around its compensation composition, tends to show the perpendicular magnetization. The RE content of the GdFeCo layer was selected richer than that of the compensation

Table 1 Typical magnetic properties of each magnetic layer.

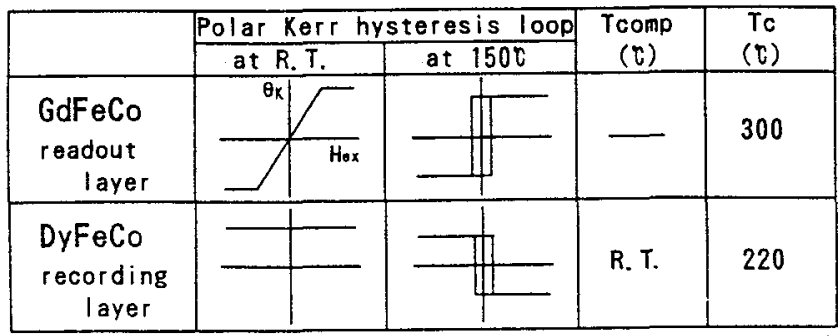

R. T. (Room Temperature) composition at the room temperature so that its magnetization direction is in-plane at the room temperature and becomes perpendicular at a higher temperature. On the other hand, the magnetization direction of the DyFeCo recording layer is perpendicular at from the room temperature to the Curie temperature. Here, the RE content of the DyFeCo layer was set around that of the compensation composition at the room temperature.

We combined these magnetic films and examined the magneto-optical properties.

Figure 2 shows the structure of the medium prepared on a glass substrate. The thickness of both magnetic layers is $40 \mathrm{~nm}$. These magnetic layers are sandwiched with AIN protective layers.

Figure 3 shows polar Kerr hysteresis loops measured from the glass side at two different temperatures. Figure 3 (a) shows the loop at $25^{\circ} \mathrm{C}$. The polar Kerr rotation angle $(\theta \mathrm{k})$ is almost zero

Glass substrate

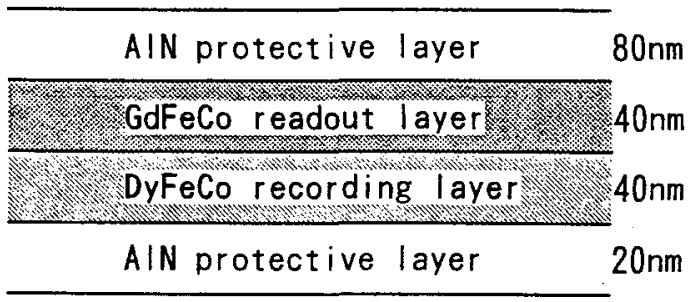

Fig. 2 Structure of a mediun in this study.

when an external magnetic field is zero. Figure 3 (b) shows the loop at $150^{\circ} \mathrm{C}$. The easy axis of the magnetization direction changes to perpendicular. The loop at $150^{\circ} \mathrm{C}$ traces reversely in comparison with those of normal RE-TM films. (Arrows in figure 3 (b) indicate the trace direction.) RE magnetic moments are dominant in the readout layer and TM ones are dominant in the recording layer at the temperature. The loop at $150^{\circ} \mathrm{C}$ shows that the magnetization direction of the readout layer follows that of the recording layer when the external field is below $\mathrm{Hx}$ in figure 3 (b). The $\mathrm{Hx}$ corresponds to the exchange coupling force between magnetic layers.

Figure 4 shows the $\theta \mathrm{k}$ of the readout layer as a function of temperature. Here, $\theta \mathrm{k}$ is the value when an external magnetic field is zero. The $\theta \mathrm{k}$ rapidly increases at between $80^{\circ} \mathrm{C}$ and $100^{\circ} \mathrm{C}$. In other words, the magnetization direction of the 
readout layer drastically changes from in-plane to perpendicular at between these temperatures.
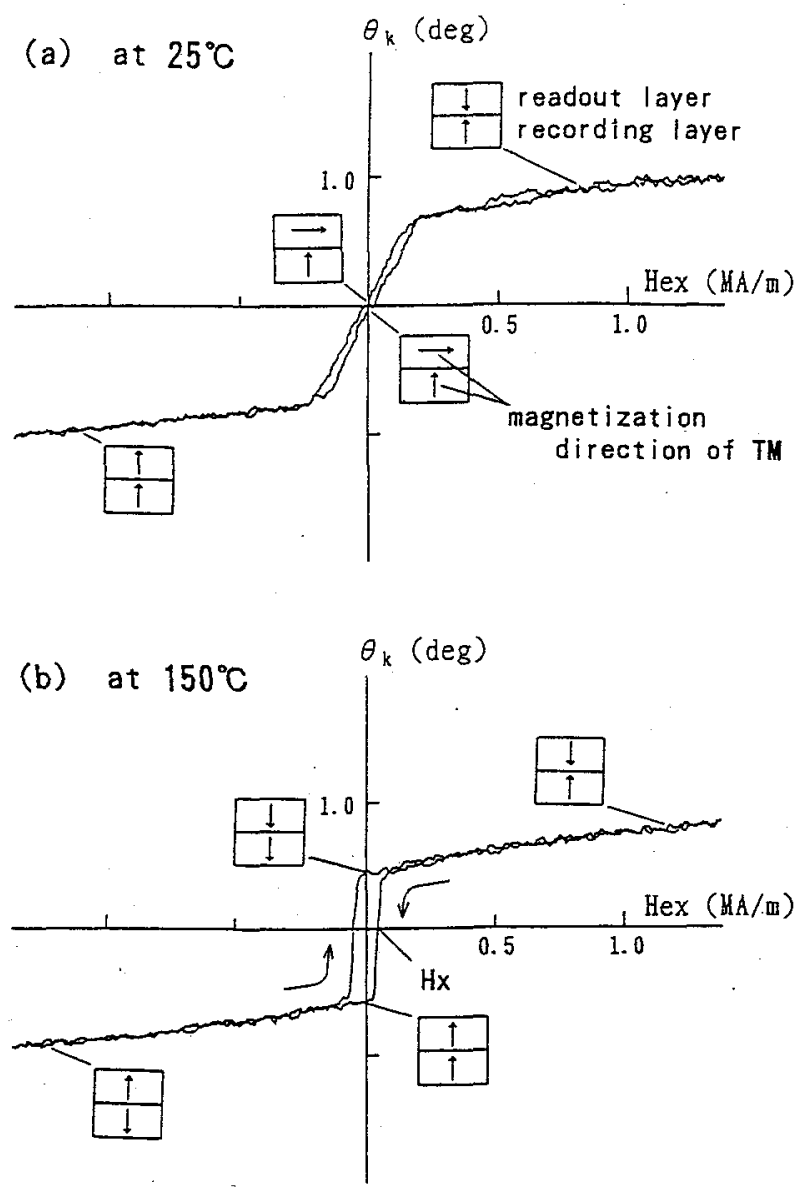

Fig. 3 Polar Kerr hysteresis loops of double magnetic layers, neasured fron the GdFeCo readout layer side at two different temperatures: (a) at $25^{\circ} \mathrm{C}$ and $(b)$ at $150^{\circ} \mathrm{C}$

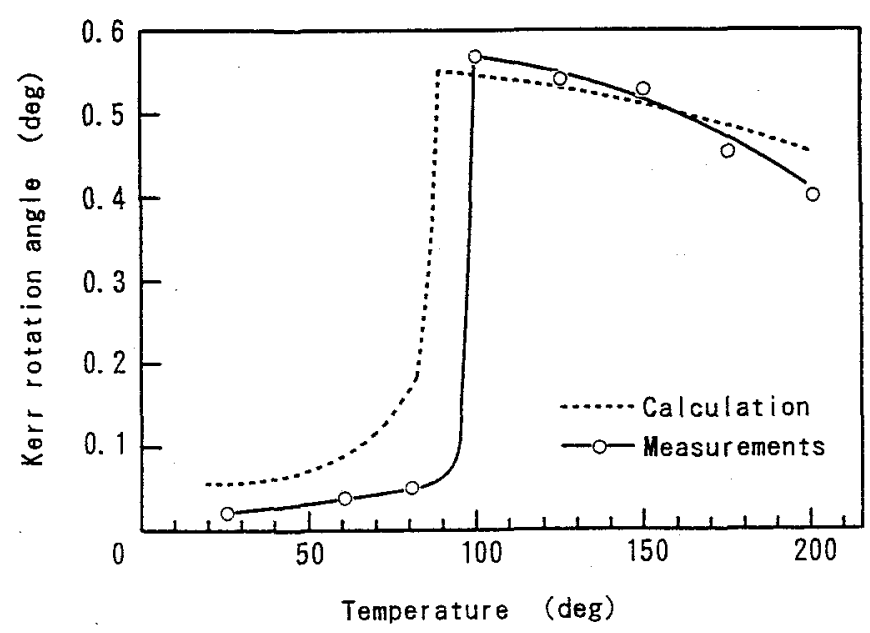

Fig. 4 Temperature dependence of Kerr rotation angle $\left(I_{k}\right)$ of double magnetic layers, measured from the GofeCo readout layer.
Figure 4 also shows a calculated value of $\theta \mathrm{k}$. In the calculation, firstly magnetic moment directions for each temperature were calculated [2] using the model developed by Andrä [3]. Next, assuming that magneto-optical effects of divided thin sheets were proportional to the perpendicular component of the TM magnetic moments, the temperature dependence of $\theta \mathrm{k}$ was calculated. The calculated dotted line agrees with the measured one.

\section{Dynamic Characteristics}

for super resolution

We investigated the dynamic characteristics of the medium formed on a glass substrate. The glass substrate had $0.8 \mathrm{um}$-width and $70 \mathrm{~nm}$-depth grooves in $1.6 \mu \mathrm{m}$ pitch which were directly formed on the disk surface by a reactive ion etching method [4]. A conventional optical head was used for the measurement, the laser wavelength was $780 \mathrm{~nm}$ and the N.A. of the objective lens was 0.55 . The linear velocity was $5 \mathrm{~m} / \mathrm{sec}$.

Figure 5 shows the read laser power dependence of the readout signal amplitude. The amplitudes are normalized with that of $0.5 \mathrm{~mW}$ read power. The length of recorded marks is $0.765 \mu \mathrm{m}$. The conventional medium which consists of AIN, DyFeCo, AIN and AI reflective layers [5] was also measured for comparison. The signal amplitude of the conventional disk is not proportional to the read power because the magnetization is reduced and the Kerr. rotation angle decreases as a temperature rises. However, the signal amplitude

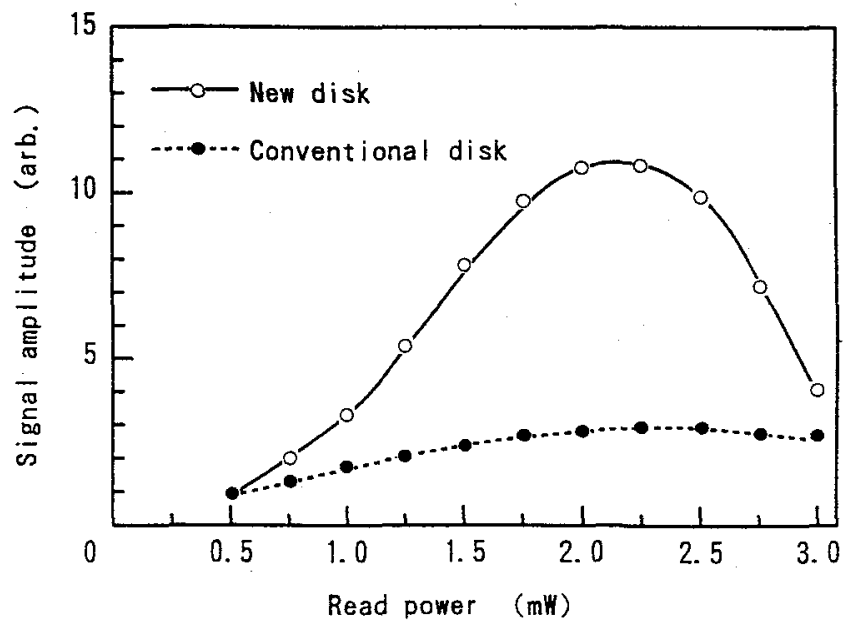

Fig. 5 Read power dependence of signal anolitude. The value of anplitudes are normalized with that of $0.5 \mathrm{ml}$ read porer. The conventional disk means a current $\omega$ disk wich consists of substrate/AIN /DyFeCo/AIN/AI. 
of the new medium sharply increases as the read power increases and the maximum signal amplitude is obtained at around the $2.25 \mathrm{~mW}$ read power. The same tendency was obtained for different sizes of recorded marks like $0.50 \mathrm{\mu m}$ and $0.35 \mathrm{\mu m}$. With the new medium, MO signal can hardly be detected when the read power is low. This is because the magnetization direction of the readout layer is in-plane. On the other hand, MO signal gets smaller when the read power is higher than $2.25 \mathrm{~mW}$. This is because the $\theta \mathrm{k}$ of the readout layer gets smaller because the temperature gets near the Curie temperature.

Figure 6 shows the mark length dependence of the carrier to noise ratio (CNR). The read power was set at $2.25 \mathrm{~mW}$ for the new disk and $1.0 \mathrm{~mW}$ for the conventional disk. The CNR of the new disk is noticeably improved in comparison with the conventional disk especially below $0.5 \mu \mathrm{m}$ mark length. The CNR of the conventional disk is almost zero for $0.35 \mu \mathrm{m}$ mark length which corresponds to the spatial cut-off frequency decided by the employed optics.

These readout results show that higher linear density is achieved using the new medium.

Next, we evaluated the cross-talk to confirm the possibility of higher track density. Generally, the cross-talk is evaluated between neighboring recorded lands, or neighboring recorded grooves in the current MO disks. However, we evaluated the value between $a$ land and its adjacent grooves. It means that recorded marks pitch in the radial direction was $0.8 \mu \mathrm{m}$ in this study.

Figure 7 shows the results of the cross-talk measurement. The value of the new disk is $-30 \mathrm{~dB}$ and it is good enough for practical use. This results show that track density can be doubled using the new disk in comparison with the conventional MO disks which have 1.6um pitch tracks.

\section{CONCLUSIONS}

Super resolution readout was realized using a new GdFeCo readout layer and a DyFeCo recording layer. The magnetization direction of the readout layer is in-plane at a room temperature and gets perpendicular at high temperatures corresponding to the central region of the light spot when the readout laser beam irradiates. Using this readout layer, higher linear density and track density compared with the conventional MO disks were achieved without an external magnetic field for readout and with a conventional optical head.

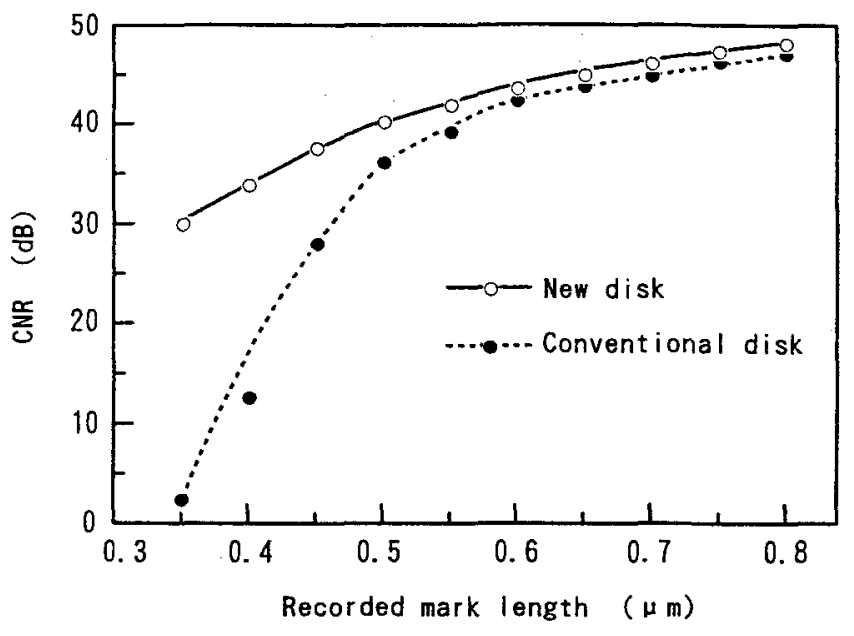

Fig. 6 Recorded mark length dependence of carrier-tonoise ratio (CNR).

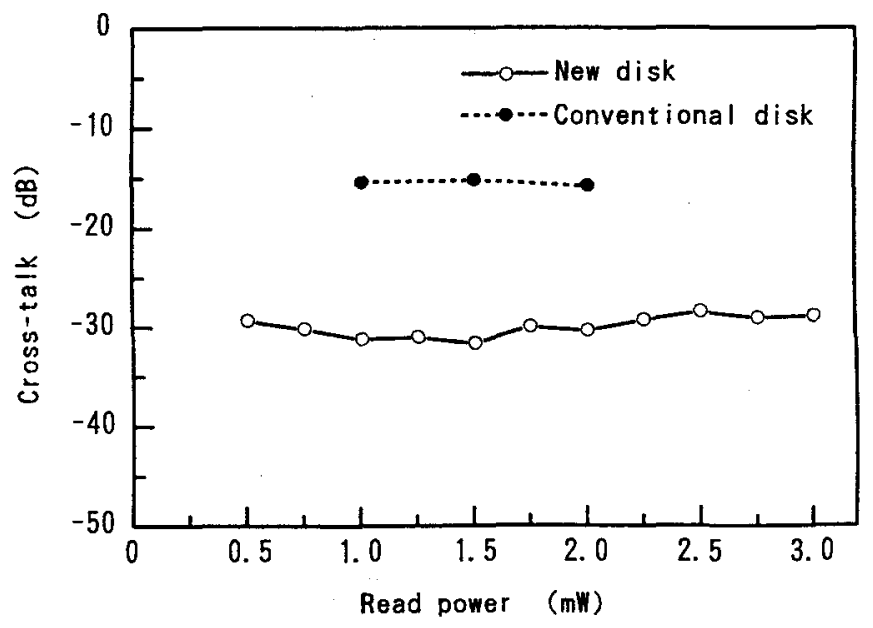

Fig. 7 Read power dependence of cross-talk. The values of cross-talk in this study was evaluated between $a$ land and its adjacent grooves. Recorded marks pitch in the radial direction was $0.8 \mathrm{~km}$ and marks length was $0.765 \mathrm{~m}$.

\section{REFERENCES}

[1]K.Aratani, A.Fukumoto, M.Ohta, M.Kaneko and K.Watanabe : Proc. SPIE, Vol.1499, Optical Data Storage Topical Meeting, Colorado Springs, p.209 (1991)

[2]J.Nakajima, Y.Murakami, A.Takahashi, K.Onta and T.Ishikawa : Digests of the 16th Annual Conference on Magnetics in Japan, 9pG-15 p.435 (1992)

(in Japanese)

[3]W.Andrä, IEEE Trans.Magn., Mag-2(3),560 (1966)

[4]T.Inui, J.Hirokane, K.Ohta, H.Yamaoka and T.Ishikawa : Proc.SPIE, Vol.1078, Optical Data Storage Topical Meeting,Los Angeles, p.204 (1989)

[5]Y.Murakami, A.Takahashi, K.Ohta and T.Ishikawa: Proc.Magneto-Optical Recording International Symposium, Tokyo, p.327 (1991) 